\title{
Serum immunoregulatory proteins as predictors of overall survival of metastatic melanoma patients treated with ipilimumab
}

\author{
Yoshinobu Koguchi ${ }^{1 *}$, Helena Hoen ${ }^{1}$, Shelly Bambina', Michael Rynning ${ }^{2}$, Richard Fuerstenberg ${ }^{2}$, Zipei Feng ${ }^{1}$, \\ Bernard Fox', Carlo Bifulco ${ }^{1,3}$, Brendan D Curti ${ }^{3,4}$, Walter Urba', Christina Milburn ${ }^{5}$, Alan J Korman ${ }^{5}$, \\ Keith S Bahjat ${ }^{1,3,6}$
}

From 30th Annual Meeting and Associated Programs of the Society for Immunotherapy of Cancer (SITC 2015) National Harbor, MD, USA. 4-8 November 2015

\section{Background}

Treatment with ipilimumab improves overall survival (OS) in patients with metastatic melanoma. Because ipilimumab targets $\mathrm{T}$ lymphocytes and not the tumor itself, efficacy may be uniquely sensitive to immunomodulatory factors present at the time of treatment.

\section{Methods}

We analyzed serum from patients with metastatic melanoma (247 of $273,90.4 \%)$ randomly assigned to receive ipilimumab or gp100 peptide vaccine (NCT00094653). We quantified candidate biomarkers at baseline and assessed the association of each with overall survival using univariate and multivariate analyses. Results were confirmed in an independent cohort of similar patients (48 of 52, 92.3\%) treated with ipilimumab (NCT00495066).

\section{Results}

Univariate analysis of biomarkers identified chemokine (C-X-C motif) ligand 11 (CXCL11) and soluble MHC class I polypeptide-related chain A (sMICA) as potential predictive biomarkers for ipilimumab but not gp100 therapy for metastatic melanoma. After controlling for baseline covariates, elevated CXCL11 and sMICA were associated with poor OS in ipilimumab-treated patients ( $\log 10$ CXCL11: hazard ratio (HR), 1.88 ; 95\% CI, 1.14 to 3.12; $P=0.014$; and $\log 10$ sMICA quadratic effect $P=$ 0.066; sMICA ( $\geq 247$ vs < 247): HR, 1.75 ; $95 \%$ CI, 1.02 to 3.01 ) but not in gp100-treated patients. Multivariate analysis of an independent ipilimumab-treated cohort confirmed the association between $\log 10$ CXCL11 and OS (HR, 3.18; 95\% CI 1.13 to 8.95 ; $P=0.029$ ), while sMICA was less strongly associated with OS $(\log 10$ sMICA quadratic effect $P=0.16$; sMICA $(\geq 247$ vs $<$ 247): HR, 1.48; $95 \% \mathrm{CI}, 0.67$ to 3.27 ).

\section{Conclusion}

Low baseline CXCL11 and sMICA were associated with improved OS in patients with metastatic melanoma after ipilimumab treatment but not vaccine treatment. Thus, pretreatment CXCL11 and sMICA may represent predictors of survival benefit after ipilimumab treatment as well as therapeutic targets. Furthermore, their role in recruiting $\mathrm{T}$ regulatory cells (CXCL11) and inhibiting cytolytic effector cells (sMICA) suggests that combination therapies targeting these molecules may synergize with CTLA-4 blockade in patients.

\section{Authors' details}

${ }^{1}$ Earle A. Chiles Research Institute, Portland, OR, USA. ${ }^{2}$ R\&D Systems, Minneapolis, MN, USA. ${ }^{3}$ Providence Cancer Center, Portland, OR, USA. ${ }^{4}$ Providence Portland Medical Center, Portland, OR, USA. ${ }^{5}$ Bristol-Myers Squibb, Redwood City, CA, USA. ${ }^{6}$ Robert W. Franz Cancer Research Center, Portland, OR, USA.

Published: 4 November 2015

doi:10.1186/2051-1426-3-S2-P96

Cite this article as: Koguchi et al:: Serum immunoregulatory proteins as predictors of overall survival of metastatic melanoma patients treated with ipilimumab. Journal for ImmunoTherapy of Cancer 2015 3(Suppl 2):P96.

${ }^{1}$ Earle A. Chiles Research Institute, Portland, OR, USA

Full list of author information is available at the end of the article 